\title{
Research on Computer Network Information Security and Its Protection Countermeasures
}

\author{
Liejuan Yang Changping Wang
}

\begin{abstract}
With the development of science and technology and the continuous development of information technology, computer network provides people with more channels of information and brings great convenience to life, computer network technology is frequently used by people, but the problem of computer network security has become very prominent and even needs to be solved urgently. Network security also brings a lot of troubles to people, and poses a great threat to people's information security. In the global scope, both developed and developing countries are facing the problem of computer network information security. The paper first analyzes the computer network information security risks and existing security problems, and puts forward corresponding protective countermeasures, and applies the formulated reasonable system to the use of computers, so that China's computer network information security can be effectively guaranteed.
\end{abstract}

\section{Keywords}

computer; network; information security; protect

\section{计算机网络信息安全及其防护对策研究}

\section{杨列娟 王昌平}

\section{摘 要}

随着现如今科技逐渐发达, 信息技术也在不断地发展, 计算机网络为人们提供了很多信息渠道, 给生活带来很大的便利, 计 算机网络技术被人们频繁地使用, 但是计算机网络安全的问题也变得非常突出甚至亟待解决。网络安全也给人们带来了很多 的困扰, 给人们的信息安全带来了很大的威胁, 在全球范围内, 不论是发达国家还是发展中国家, 都面临着计算机网络信息 安全问题。论文先对计算机网络信息安全风险和所存在的安全问题进行了分析, 并且提出了相应的防护对策, 将制定的合理 制度运用到计算机使用中，使中国的计算机网络信息安全能得到有效地保障。

\author{
关键词 \\ 计算机; 网络; 信息安全; 防护
}

\section{1 引言}

在网络信息社会, 计算机技术在很多领域都占有重要地

位。但是在计算机运用和网络信息传递过程中, 不可避免地 遇到各种各样的网络安全问题, 网络漏洞也在频繁发生, 很 多用户信息被泄露, 重要数据被盗取破坏。因此, 加强计算 机网络安全问题已经成为政府机构最重视的问题之一。论文 重点研究了存在计算机信息安全隐患的几个方面, 并结合相

【作者简介】杨列娟（1984-），女, 中国陕西西安人, 研究 生学历, 工程师, 从事网络安全研究。

王昌平 (1984-), 男, 中国甘肃定西人, 研究生学历, 工程师, 从事网络安全研究。
关的技术改革创新要求, 制定对计算机网络安全行之有效的 解决方案。

\section{2 计算机网络信息安全的特点}

在当今这个互联网飞速发展的时代中, 计算机网络的发 展非常迅速。因为有网络的存在, 大量的信息将会很容易被 查找到, 所以我们现在更应该注重网络信息安全方面的一系 列问题。

在计算机网络信息安全中有三个主要的特点: 一是要时 刻保证计算机网络信息在传输和储存方面的完整性, 避免出 现信息丢失和被破坏的问题和信息的安全性; 二是要注重保 密性, 用户和预览网络信息时, 需要得到相关的授权才能让 
其使用; 三是在网络中双方进行相互交换信息时要有精准的 身份认证，这样就可以使所传递的信息得到可靠的保证。

\section{3 当前计算机网络信息安全防护的情况}

随着互联网的飞速发展, 有大量的信息将会存在被泄露 的风险, 从如今的网络信息安全防范的情况来看, 各种网络 安全问题频发。一方面没有合理的安全防护, 另一方面计算 机网络存在脆弱性, 网络信息还会因为一些自然原因而导致 安全问题的发生。当然, 在计算机网络信息安全的防护中还 有一点尤为重要, 那就是要时刻对网络病毒的攻击进行监控 和防护, 计算机就如同人体一样, 也会遭受各种各样的病毒 侵扰, 这很容易导致网络信息泄露, 从而严重影响计算机网 络信息的安全性。因此, 我们要加大对网络信息安全防护的 力度, 让每一位使用网络的用户都能得到更加安全放心的信 息保障 ${ }^{[1]}$ 。

\section{4 加强计算机网络信息安全防护的技术}

\section{1 防火墙技术}

防火墙之中的 NAT 防御软件是通过 IP 地址转换成临时 注册的 IP 地址。内部网访问外部网时, 一旦成功通过安全网 卡，那么此防火墙就会自动把源地址同端口伪装之后与外部 相连。用户们通过对网络数据的访问和传输, 控制信息输入 的端口, 这就是技术效果。技术还可以过滤用户不需要的知 识内容, 可以验证信息网络, 也可以对用户们访问做出服务 要求。在用户在外连公共网上网时, 可通过防火墙技术进行 隔离某些病毒。设计人员还可以通过防火墙来对计算机网络 的 IP 地址和 USB 数据接口进行准确的分析和探究, 可以将 网络中的信息资源识别展现出来, 可以将网络传输的信息资 源和设计人员共同设定的网络访问数量表, 来对不同的数据 进行调查研究, 通过对数据输入和输出的高效控制, 防火墙 技术过滤与整个系统设备不协调的系统内容。此外，还可以 利用高科技的技术水平来对不明攻击的病毒进行防护，从而 可以保护计算机网络信息资源的安全性。防火墙技术还可以 在用户联都公共网的时候, 通讯时信息内容遭到泄露时可对 其进行保护作用。如果内部网络用户遭到外部用户的强行访 问，这时防火墙技术就会分析外部用户的数据信息资源是否 完整, 还会探究其中的病毒问题, 还会䇥选其中不符合要求 的数据信息, 在内部用户同意的情况下才可以让外部用户进
行连接使用, 这样才可以保证计算机网络整体的信息安全 ${ }^{[2]}$ 。

\section{2 数据加密技术}

网络加密技术是指发送信息的这个人先对信息进行加密 处理, 接触加密的密码由接收信息这方掌握。接收方接收信 息之后就使用密码解除信息的加密状态, 这样也就保证此次 信息传输安全的完成。简而言之, 加密技术也就是利用的密 钥以保证信息在传输过程中的安全性。在用户使用计算机网 络技术的时候, 也可以通过数据加密技术, 可以融合网络信息, 这样可以保护用户信息的安全性。由于网络信息在不断的高 速发展，在各个领域不断的被使用，所以用户信息的安全问 题越来越严重, 影响用户个人信息的安全因素也越来越多。 在这种问题以下, 就要求技术人员对信息资源数据进行加密, 并将加密技术运用到防护网络信息中, 如果用户在某些网站 上登录自己的信息时，应该通过加密系统，来对个人信息进 行隐蔽, 保证不会被泄露。在用户使用过该网站过后退出登 录的时候, 系统也不应该留下任何痕迹, 也是为了防止用户 的个人信息遭到盗取 ${ }^{[3]}$ 。

\section{3 审计跟踪技术}

计跟踪（audit trail）, 是系统活动的流水记录。该记录 按事件从始至终的途径, 顺序检查审计跟踪记录、审查和检 验每个事件的环境及活动。在用户使用计算机网络系统, 在 整个流程中系统可根据用户使用的记录检查内容, 对其内容 进行监控一系列的操作。对系统的跟踪技术也可以防止其他 网络对其网络的入侵, 也可以解决自身网络所出现的漏洞。 技术管理员可对网络进行安全审查和监视，提升整个信息资 源的完整性。在检测计算机时, 如果网络数据出现一些差错, 漏洞扫描仪可以检测出来, 可以试试正确的补救方法, 将计 算机的内部与外部恢复正常, 也可以做好病毒入侵网络的提 前防护工作。

\section{5 加强计算机网络信息安全防护的管理措施}

\section{1 增强用户安全防范意识}

在当今网络用户飞快增长的过程中, 很多用户都想要追 求更高的网络速度, 在使用网络的时候会通过关闭一些防护 软件来提高网速, 这样一来, 虽然会让网速有所提高, 但也 使计算机暴露在病毒侵害的环境中。所以在使用网络的同时, 用户要多加注意网络信息安全的防护, 要不断增强自身的网 
络安全防范意识, 进行文明合理的网络使用, 这样才能让病 毒没有可乘之机。与此同时, 国家也需要做出相应的对策, 让用户得到更加安全和稳定的网络使用环境。

\section{2 强化软件硬件安全管理}

在使用计算机的同时, 也要时候关注所使用的设备有没 有出现一些安全隐患, 这样才能更加及时地对出现问题的部 件进行有效的替换, 从而进一步保证计算机网络能在更加安 全的环境中使用, 这也是非常重要的安全防护措施。我们可 以在使用硬盘等一些储存设备的同时, 对其进行更加安全的 防护处理, 这样就可以进一步降低计算机网络所面临的风险。 与此同时, 在对计算机软件进行管理和修复的时候, 要时刻 关注软件的更新动态, 这样才能让所使用的软件保持一个更 加安全的状态。我们还会通过对防毒软件的开发, 从而降低 计算机网络被病毒所侵害风险。因此, 对软件进行管理才是 整个安全防范过程的核心, 只有正确地使用和了解软件, 才 能让网络信息拥有更加安全的保障。

\section{3 落实信息安全管理工作}

在进行网络信息安全防护的过程中, 我们要对工作人员
进行更加严格的要求，要时刻加强对网络信息安全的管理， 不能掉以轻心, 以免出现难以应对的麻烦。这也需要对安全 管理的技术进行更新, 从而让整个系统更加的安全。与此同时, 还要对网络安全进行实时的监控, 这样就可以及时发现可能 会出现的问题，从而进一步降低计算机网络安全所面临的风 险。此外, 还可以通过对风险级别的评估, 来进行更加有效 的防范。

\section{6 结语}

综上所述, 为了给用户制造一个安全的计算机网络环境, 必须要在实际的情况中, 对网络信息的安全特点做到深入了 解, 要提前做好防护工作, 并且还要探究更多的防护方案, 也是为了推进中国计算机网络技术的高效发展。

\section{参考文献}

[1] 董毅.计算机网络信息安全防护 [J]. 中国战略新兴产业,2018 $(05 \mathrm{X}): 123$.

[2] 许子桓. 计算机网络信息安全防护 [J]. 数码世界, 2018(04):51.

[3] 孙苏鹏. 计算机网络信息安全防护研究 [J]. 科技传播,2018 (20):108-109. 